\title{
Problems and Solutions during the Growth of LSO Single Crystal with Czochralski Method
}

\author{
Zhou Wenping ${ }^{1,2}$ \\ School of Materials and Metallurgy ${ }^{1}$ \\ Northeastern University ${ }^{1}$ \\ College of Anergy and Power ${ }^{2}$ \\ Shenyang Engineering College ${ }^{2}$ \\ Shenyang, China \\ e-mail: zhouwp@sie.edu.cn \\ Liu Xudong \\ College of Anergy and Power \\ Shenyang Engineering College \\ Shenyang, China \\ e-mail: liuxd@sie.edu.cn \\ Niu Wei \\ College of Anergy and Power \\ Shenyang Engineering College \\ Shenyang, China \\ e-mail: flysky168@sina.com
}

\author{
Bi Xiaoguo \\ College of Anergy and Power \\ Shenyang Engineering College \\ Shenyang, China \\ e-mail: bixiaoguo@sie.edu.cn
}

Tang Jian

College of Anergy and Power

Shenyang Engineering College

Shenyang, China

e-mail: tangjian@sie.edu.cn

Sun Xudong

School of Materials and Metallurgy

Northeastern University

Shenyang, China

e-mail: xdsun@mail.neu.edu.cn

\begin{abstract}
The main problems and causes, such as crucible erosion, components volatilization, other impurities intrusion and instable scintillation properties, during the growth of cerium doped lutetium silicate single crystal with Czochralski method were discussed in this paper. According to process characteristics and principle of Czochralski method and flame fusion method, all of the problems above could be solved by flame fusion method, and a new research direction for preparation of $\mathrm{Ce}: \mathrm{Lu}_{2} \mathrm{SiO}_{5}$ single crystal was provided.
\end{abstract}

Keywords-cerium doped lutetium silicate single crystal(Ce:Lu $\left.\mathrm{Lu}_{2} \mathrm{SiO}_{5}\right)$; Czochralski method; flame fusion method; segregation coefficient; energy resolution

\section{INTRODUCTION}

Ce: LSO single crystal had excellent comprehensive scintillation properties, and was used in nuclear physics and other fields, especially in the field of nuclear medicine imaging and positron emission tomography (PET) technology ${ }^{[1-3]}$.

The Czochralski method and sol-gel method for the preparation of Ce: LSO single crystal had been reported currently ${ }^{[4-5]}$, but only Czochralski method was used in commercial production ${ }^{[2-3,6]}$. The typical characteristic of the Czochralski method compared with flame fusion method was the molten filled with the crucible. Thus, there were basic requirements for growth of crystal with Czochralski method. As following: First, Oxidative destruction of the crucible itself did not occur under growth conditions. Second, the molten raw material did not react chemically with the crucible material, which included graphite, tungsten and its alloys, platinum and iridium, etc. To ensure the chemical stability of the crucible under the growth conditions, the growth process was generally in a vacuum and inert protective atmosphere. Obviously, not all of the materials met the above conditions, such as rutile single crystal, a raw material component of the melt decomposition occurred in the condition of a vacuum or insufficient oxygen partial pressure ${ }^{[7]}$. Third, the molten composition of raw materials was maintained uniformity. Forth, the raw materials were all melted and maintained solution during the growth process of crystal with Czochralski method. Fifth, the speed of growth was slow and generally $0.5-2 \mathrm{~mm} / \mathrm{h}$. For the doped crystals, due to the removing impurity effect from the growth interface, content of doping elements in the melt increased unceasingly, and so the content of doping elements was the same distribution for grown crystals, especially for Ce:LSO crystal. In contrast, the growth of single crystals, such as rutile and strontium titanate, with flame fusion method was held a short time for molten, and supplied the deficiencies of the Czochralski method ${ }^{[7-10]}$.

Combined the physical and chemical characteristics of cerium-doped lutetium silicate crystal $\left(\mathrm{Ce}: \mathrm{Lu}_{2} \mathrm{SiO}_{5}\right)$ with the characteristics of the Czochralski and flame fusion method, The main problems and causes, such as crucible erosion, components volatilization, other impurities intrusion and instable scintillation properties, during the growth of cerium doped lutetium silicate single crystal with Czochralski method were discussed, and a new 
research direction for low-cost and commercial preparation of $\mathrm{Ce}: \mathrm{Lu}_{2} \mathrm{SiO}_{5}$ single crystal was provided.

\section{CE: LSO CRYSTAL GROWTH PROCESS}

A. Raw materials and its physical and chemical properties of Ce: LSO

Despite of the reported sol-gel method for preparation of cerium doped lutetium silicate (Ce: LSO) materials ${ }^{[4-5]}$, the Ce:LSO crystal growth raw materials were solid-phase synthesized by high-purity $(99.99 \%)$ of $\mathrm{Lu}_{2} \mathrm{O}_{3}, \mathrm{SiO}_{2}$ and $\mathrm{CeO}_{2}{ }^{[11-12]}$. The proportions of the fired raw materials were weight according to the following formula:

$$
\mathrm{SiO}_{2}+(1-\mathrm{x}) \mathrm{Lu}_{2} \mathrm{O}_{3}+2 \mathrm{xCeO} \mathrm{Cu}_{2}=\mathrm{Lu}_{2(1-\mathrm{x})} \mathrm{Ce}_{2 \mathrm{x}} \mathrm{SiO}_{5}
$$

In the formula, the $\mathrm{x}$ was the Ce mole fraction of atoms in the molten $(\mathrm{x}=0.25 \%, 0.5 \%, 1.0 \%$, etc. $)$; the doping concentration in the crystal was the product of $\mathrm{x}$ and the segregation coefficient. After weighing and grinding, the raw materials were mixed evenly, and pressed into blocks in the hydraulic press. The blocks were sintered at $1000 \sim$ $1200{ }^{\circ} \mathrm{C}$ under solid phase reaction, and stored in the drying box. The physical and chemical properties of Ce: LSO raw materials and its components were shown in TABLE I.

TABLE I. THE PHYSICAL AND CHEMICAL PROPERTIES OF CE: LSO RAW MATERIALS AND ITS COMPONENTS

\begin{tabular}{|c|c|c|c|c|}
\hline Material & $\mathrm{Ce}: \mathrm{LSO}$ & $\mathrm{SiO}_{2}$ & $\mathrm{Lu}_{2} \mathrm{O}_{3}$ & $\mathrm{CeO}_{2}$ \\
\hline property & & & & \\
\hline Melting point $\left({ }^{\circ} \mathrm{C}\right)$ & $2050-2070$ & 1710 & $2467-2510$ & $2400-2600$ \\
\hline Boiling point $\left({ }^{\circ} \mathrm{C}\right)$ & - & 2230 & 3980 & 3500 \\
\hline
\end{tabular}

\section{B. Problems and causes of growth of Ce: LSO single crystal with Czochralski method}

Crucible corrosion was the first problem of Ce: LSO crystal growth with Czochralski method. The Ce: LSO was a high-temperature oxide crystal, and its components had high melting point listed in TABLE I. The crystal growth process needed oxidizing atmosphere. The growth of crystal with Czochralski method was generally in a metal iridium crucible in which $\mathrm{Lu}_{2} \mathrm{O}_{3}$ was decomposed to free oxygen molecules at such high temperatures even if there was no oxygen in the growth environment. In order to suppress the oxygen free molecules into the environment, the oxygen partial pressure in the atmosphere usually was increased, which induced to exacerbate iridium crucible oxidation. In this case, particularly iridium crucible was volatile or ablation into the melt, which not only increases the cost of the growth, but also the iridium particles was easily volatilized into the crystal and formatted the light scattering centers ${ }^{[11]}$.

The second problem was that the melting points had a big difference among $\mathrm{SiO}_{2}, \mathrm{Lu}_{2} \mathrm{O}_{3}$ and $\mathrm{CeO}_{2}$ listed in table 1, which induced the problems as following: The first one was that the formulation of a single-phase Ce: LSO structure was difficult after the calcinations process, because of the difficult diffusion of high melting point
$\mathrm{CeO}_{2}$ and $\mathrm{Lu}_{2} \mathrm{O}_{3}$. The free $\mathrm{Lu}_{2} \mathrm{O}_{3}$ and $\mathrm{CeO}_{2}$ particles was probably present in the formed Ce:LSO matrix structure. The second one was that the $\mathrm{Lu}_{2} \mathrm{O}_{3}$ and $\mathrm{CeO}_{2}$ component with high melting point did not probably melt and was granular retention in the formed melt, which induced the inclusions in the resulting crystals. The third one was that the component $\mathrm{SiO}_{2}$ with low melting point was easily volatile, which lead to changes in the composition of the melt, and was difficult to prepare the high-quality crystals.

The third problem was that the densities(listed in table 1) were different among the $\mathrm{SiO}_{2}, \mathrm{Lu}_{2} \mathrm{O}_{3}$ and $\mathrm{CeO}_{2}$, which lead to the different melt composition in the height direction, for example the $\mathrm{SiO}_{2}$ with the smaller density component floated above the melt, and the poor performance of the crystal.

The fourth problem was that the small segregation coefficient of $\mathrm{Ce}$, about 0.20 to 0.25 , induced the $\mathrm{Ce}$ ion concentration was much higher in top of LSO crystal than that of bottom, which led to light output was 2 to 5 times at the top of the bottom, and the decay time was $41 \mathrm{~ns}$ to $50 \mathrm{~ns}$. Meanwhile, the energy resolution had greater changes, and the differences measured in different laboratories varied from $7 \%$ to $17 \%$, or even worse.

These factors led to complexity and performance instability of Ce: LSO crystal single crystal prepared by Czochralski method. Dissolution of the crucible led to the contamination of the crystal, and increased the cost of crystal. This problem could be avoided with other crystal growth methods without crucible. In the Ce: LSO composition, although there was big difference melting point of $\mathrm{SiO}_{2}, \mathrm{Lu}_{2} \mathrm{O}_{3}$ and $\mathrm{CeO}_{2}$, but if the low melting component was less volatile, and the melt state remains short of time during the growth process, then the resulting substantially reduced the harm. Likewise, if a crystal was grown directly on the melted material, and maintained in the melt state for shorter time, the degree of stratification of the components was greatly reduced. Despite the fact that the melt shortening retention time can't eliminate the harm during to the small segregation coefficient, improving the growth rate can still reduce its harm.

\section{Analysis on the basis of flame fusion growth of Ce: LSO single crystal.}

In view of the above problems in the growth of $\mathrm{Ce}$ : LSO single crystal with the Czochralski method, the flame fusion method was proposed, which had not been reported. Compared with the Czochralski method, the flame fusion method was a method for preparation of single crystal from melt too, and there was no crucible containing the melt during growth of crystal. The raw material powder melt at high temperature of the oxyhydrogen flame, and the melt dripping drop directly on the seed crystal or the crystal for a short time. Therefore, the flame fusion method for preparation of Ce: LSO crystal avoid the problems encountered for Czochralski method.

(1) Crucible was not required during the crystal growth with flame fusion method, and the crystal growth process was carried out in an oxidizing or reducing atmosphere in according to the melt properties of growth crystals. Therefore, there was not iridium crucible corrosion and other problems during the growth of $\mathrm{Ce}$ : LSO single crystal.

(2) Oxyhydrogen flame was often used in the flame 
fusion method for preparation of single crystal, such as corundum gemstone, rutile, and strontium titanate. The center temperature of the oxyhydrogen flame can reach $2800{ }^{\circ} \mathrm{C}$, and the temperature gradually lowered from the center to ambient. It was normal that Ce: LSO raw materials and component instantly melt by reasonable design of burner, chamber and the growth position. Due to the oxygen partial pressure could be controlled and the melt was held for a short time, as shown in Fig .1, the possibility of $\mathrm{SiO}_{2}$ evaporation loss greatly reduced. Meanwhile, the existence of solid-phase inclusions and other issues caused by the unmelted $\mathrm{Lu}_{2} \mathrm{O}_{3}$ and $\mathrm{CeO}_{2}$ component could be eliminated in crystal.

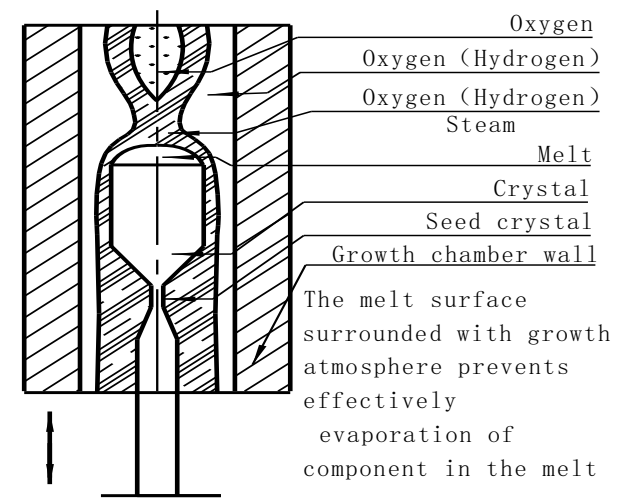

Figure 1. Scheme of crystal growth with flame method

(3) Stratification of the melt caused by the different densities of components in the Ce: LSO single crystal could be well suppressed by the flame fusion method, as shown in Fig .1. The degree of component stratification of the melt was proportional to the maintained time of state and the melt height. The melt height was set $h$, the melt density of $\mathrm{SiO}_{2}$ was $\rho$ and the floating speed was $\mathrm{v}$, and component was uniform when time was $t_{0}$. When the time was $t_{1}$, the distribution of low density of $\mathrm{SiO}_{2}$ along the $\mathrm{Y}$ direction was:

$$
\frac{\partial C_{S i O_{2}}}{\partial y}=\rho v\left(t_{1}-t_{0}\right), \quad 0 \leq y \leq h
$$

The rate of crystal growth with flame fusion method was generally $10 \mathrm{~mm} / \mathrm{h}$, while the rate of crystal growth with Czochralski method was generally $0.5-1.0 \mathrm{~mm}$, which was seen by Equation 1. The degree of stratification of the melt during crystal growth by Czochralski method was 10 times more than that of flame fusion method, so the flame fusion can effectively suppress delamination problems of melt.

(4) The growth rate of Ce: LSO single crystal by flame fusion method is about 10 times slower than that of Czochralski method, which could be seen that the speed of growth interface to melt was about 10 times slower. Since segregation coefficient of Ce was between 0.22-0.25, and the effective segregation coefficient is less than 1 . In other factors (boundary layer thickness, etc.) remain the same circumstances, with the accelerated speed of advancing solid-liquid interface, the effective segregation coefficient also increases ${ }^{[13]}$, which reduced uneven distribution of $\mathrm{Ce}$ in Ce: LSO single crystal and effectively solved unstable performance problems caused by $\mathrm{Ce}$ segregation coefficient during the growth process of Ce: LSO single crystal with Czochralski method.

\section{Dislocation of crystal growth by flame fusion method}

Crystal growth by flame fusion method also had shortcomings, and the main problem was the large temperature gradient and fast growth speed, which led to high dislocation density in the grown crystals. However, dislocation had no effect on the flashing light of certain crystals. For example, forging $\mathrm{NaI}(\mathrm{Tl})$ crystal was a rough single crystal, and was made by plastic deformation at a certain temperature and pressure. After plastically deformed, the single crystal had some constant interaction and proliferation of dislocation, and formed multilateral and sub grain structure, which improved the characteristics of the original crystal easy to cleavage by the edge (100) of surface and enhanced the ability of its resistance to thermal shock and mechanical vibration. But flashing performance was not affected. Furthermore, more complex geometries and large-sized crystals, such as hexagonal, square, rectangular, etc. and a length of more than $200 \mathrm{~mm}$ crystals, were readily prepared by hot forging process. Currently forging $\mathrm{NaI}(\mathrm{Tl})$ had been widely used in the field of space research, oil well logging, geological exploration and nuclear medicine etc.

\section{THE COMPARISON OF FLAME FUSION GROWN SINGLE CRYSTALS OF RUTILE AND STRONTIUM TITANATE AND THIS PROGRAM}

For the two kinds of single crystals of rutile (TiO2) and strontium titanate ( $\mathrm{SrTiO} 3)$, the melting point is equivalent to Ce: LSO single crystal, at about $1855{ }^{\circ} \mathrm{C}$ and $2060{ }^{\circ} \mathrm{C}$ respectively, not suitable grown by Czochralski method in the crucible. In the past decade, in order to produce high quality single crystal, the project team carried out experiments to grow rutile and strontium titanate with flame fusion method [7-10]. By the reasonable control of the furnace atmosphere,growth rate, temperature gradient and annealing conditions, a $\varnothing 30 \mathrm{~mm} \times 50 \mathrm{~mm}$ rutile single crystal and a $\varnothing 30 \mathrm{~mm} \times 60 \mathrm{~mm}$ strontium titanate single crystal were produced. The resulting crystals in terms of integrity, transmission, etc. have reached commercial standards. Practice has proved that flame fusion method for single crystal of high melting point and not suitable for growth in crucible is a good choice.

\section{CONCLUSION}

The Ce: LSO single crystal prepared by the Czochralski method had some problems, such as crucible dissolution, the volatile component, impurity immersed and scintillation unstable performance etc, because of the high melting point and the density difference large between the components. By comparing the similarities and differences between Czochralski and flame fusion method for preparation of the Ce: LSO crystal, reasons for the existence of these problems induced by Czochralski method had been elaborated, which was effectively solved by the latter method. The flame fusion method for the growth of single crystal provided a new research direction. 


\section{REFERENCES}

[1] Zhang M R, Wei J, "Status of research and development on crystals with properties of high density and fast decay time," Joural of the Chinese ceramic society, vol. 32,pp384-391, March 2004.doi:ttp://www.gxyb.cbpt.cnki.net/WKC/WebPublication/ pap erDigest.aspx?paperID=4AA0D59D-4C65-4B27-99A9-22B3F 38 A4D18.

[2] LECOQ P, “The high energy physics demand for a new generation of scintillators" , Journal of Luminescence, vol. 60, pp 948 955. January1994. doi:10.1016/0022-2313(94)90318-2.

[3] MOSES W W, DERENZO S E, "Proceedings of the International Conference on Inorganic Scintillators and Their Applications” ,Delft Netherlands ,1996,pp9 -16.

[4] Iwanczyk J S, Patt B E, Tull C R,et al., " New LSO Based Scintillators” , IEEE.Trans.Nucl.Sci. vol. 47, June 2000,pp17811786.

[5] Bescher, E.; Robson, S. R.; Mackenzie, J. D.; Patt, B.; Iwanczyk, J.; Hoffman, E. J.," New Lutetium Silicate Scintillators", J. Sol. Sci. Tech, vol.19,December 2000,pp325-328,doi: 10.1023/A:100878 5616233

[6] Qin Lai-shun , Ren Guo-hao,"Progress and Prospect in the Development of LSO Scintillation Crystal", Ournal of synthetic crystals, vol. 32,August 2003,pp.286-293, doi: http://cnki.lib. sie.edu.cn/kns50/detail.aspx?QueryID $=126 \&$ CurRec $=1$.

[7] Bi X G, Xiu Z M, Ma W M, Sun X D," Study on growth of rutile(TiO2) single crystals",Journal of Northeastern
University(Natural Science),Vol.25,Oct.2004,pp.977-979, doi http://xuebao.neu.edu.cn/natural/CN/volumn/volumn_1300.shtml.

[8] Bi X G, Xiu Z M, Ma W M, Sun X D, Zhao H S, Cao Z J, Guo G Y, Xiao J T,"Experimental Study on Growth Conditions of BigBulk Rutile Crystal",Journal of Synthetic Crystals. Vol.33, 2004,pp.244-249, doi:http://cnki.lib.sie.edu.cn/kns50/detail.aspx? QueryID=27\&CurRec $=1$.

[9] Bi Xiaoguo, Liu Xudong, Niu Wei, "Flame-fusion Growth of Rutile Single Crystal” , Advanced Engineering Materials II, Vol 5, 2012,pp.535-537. doi: 10.4028/www.scientific.net/AMR.535537.2571 .

[10] Bi X G, Huang F, He F M, Zhao H S, Sun X D, “Runover During the Growth of $\mathrm{SrTio}_{3}$ Crystal” , Journal of Synthetic Crystals. Vol. 34, April2005,pp.328-331, doi:http://cnki.lib.sie.edu.cn/kns50 /detail.aspx?QueryID=118\&CurRec $=1$.

[11] Qin L S, Ren G H, Li H Y, L S, P Y, "Main Problems in the Growth of Lu2SiO5:Ce Scintillation Crystals", Journal of the chinese ceramic society, vol.32,November2004, pp.1361-1366, doi: http://www.gxyb.cbpt.cnki.net/WKC/WebPublication/paperDigest. aspx?paperID=87AD915E-86D0-41AA-80AF-C7696798508C.

[12] Wu Guangzhao,Zhang Xinmin,Zhurude, "Growth of $\mathrm{Ce}^{3+}: \mathrm{Lu}_{2} \mathrm{SiO}_{5}$ Scintillation Crystals" ,Journal of Synthetic Crystals, vol.25, May1996 ,pp.175-176,doi: http://www.cnki.com.cn/Article/CJFD Total-RGJT602.017.htm.

[13] Zhang K C , Zhang L H. Crystal growth science and technology, 3rd ed. vol. 1, Beijing: Science Press, 1997,pp83-91. 